\title{
Species specificity of a novel factor which augments the expression of MHC class I antigens on tumor cell lines
}

\author{
Niti Puri, ${ }^{1}$ Aparna Raval ${ }^{1}$ and \\ Rajiv K. Saxena ${ }^{1,2}$ \\ 1 School of Life Sciences, Jawaharlal Nehru University, \\ New Delhi 110067, India \\ 2 Corresponding author
}

Accepted 3 June 1997

Abbreviations: MHC, major histocompatibility complex; MHC-AF, MHC augmenting factor; IFN- $\gamma$, interferon $\gamma$;Con-A, concanavalin-A; PBS, phosphate-buffered saline

\begin{abstract}
A novel factor which augments the expression of major histocompatibility complex I (MHC augmenting factor or MHC-AF) antigens on tumor cell lines, has been isolated from the culture supernatants of human peripheral blood mononuclear cells activated by concanavalin-A. A mouse equivalent of this factor has also been isolated from the culture supernatants of mouse spleen cells activated by mitogens or in a mixed lymphocyte reaction. Mouse MHC-AF enhances the expression of class I MHC antigens on murine tumor cell lines ( EL-4 and BW5147 ) but not on human tumor cell lines (K562 and HR-7). Human MHC-AF on the other hand enhances the MHC I expression on both human as well as murine cell lines. Interferon gamma (IFN- $\gamma$ ), a cytokine also known to enhance the expression of MHC I antigens, acts in a highly species specific manner with mouse IFN $-\gamma$ augmenting the MHC I on murine tumor cell lines and human IFN- $\gamma$ augmenting the MHC I on human tumor cell lines only. These results indicate important differences in the cross species biological activities of MHC-AF and IFN- - , and provide additional evidence for MHC-AF being distinct from IFN- $\gamma$.
\end{abstract}

Keywords: major histocompatibility complex, lymphokines, lymphocytes, interferon gamma

\section{Introduction}

Major histocompatibility complex (MHC) antigens play a central role in interactions within immune cells and between cytotoxic lymphocytes and tumor cells (Zinkernagel and
Doherty, 1979; Karre et al., 1986; Saxena, 1997). Factors which modulate expression of MHC antigens can be potent immunomodulators. Many factors including interferon (Piontek et al., 1985; Wiebke et al.,1990), phorbol esters and sodium butyrate (Dokhelar et al., 1982,1984), are known to augment class I MHC expression on tumor cells. Work from our laboratory has described a putative novel cytokine termed MHC augmenting factor (MHC-AF), which enhances the class I MHC expression on tumor cells and renders them resistant to NK lysis (Saxena, 1987; Saxena et al., 1988, 1989, 1992, 1996; Puri, 1995; Raval et al., 1997). Interferon $\gamma$ (IFN- $\gamma$ ) has activities similar to MHC-AF, and since it is also produced by mitogen activated lymphocytes, it is possible that the MHC-AF is same as IFN- $\gamma$. Biological and biochemical properties of MHC-AF, however, strongly suggest that it is distinct from IFN- $\gamma$ (Saxena et al., 1987, 1988, 1992). MHC-AF unlike IFN- $\gamma$, is resistant to $\mathrm{pH} 2$ treatment and lacks anti-viral activity (Dokhelar et al., 1984; Saxena et al., 1988). While the final proof of MHC-AF being different from IFN- $\gamma$ will come when the former is purified to homogeneity, this has posed problem since MHC-AF is produced in extremely low quantities which makes its purification difficult. We have devised purification schemes utilizing liquid chromato-graphy, isoelectric focusing and ionexchange and reverse phase HPLC steps, but MHC-AF has not yet been purified to homogeneity (Saxena, 1987; Saxena et al., 1988, 1992, 1996; Raval et al., 1997). Recently, we have isolated a MHC-AF activity from culture supernatants of murine spleen cells activated by mitogen or in a mixed lymphocyte reaction (Saxena et al., 1992; Puri, 1995). Having obtained MHC-AF preparations from two species, we have explored the species specificity of the biological activity of MHC-AF and compared it from that of IFN- $\gamma$. Our results indicate a major difference in species specificities between MHC-AF and IFN- $\gamma$.

\section{Materials and Methods}

\section{Culture medium}

Tumor cell lines were maintained in RPMI 1640 from Sigma (St. Louis, MO), supplemented with $5 \%$ fetal calf serum, $20 \mu \mathrm{M} 2$ 2-mercaptoethanol, $300 \mu \mathrm{g} / \mathrm{ml}$ glutamine, and 60 $\mu \mathrm{g} / \mathrm{ml}$ gentamicin (complete medium). For generation of $\mathrm{MHC}-\mathrm{AF}$, the lymphocyte cultures were carried out in commercially available serum free medium, DCCM-2 from Biological Industries, Israel, supplemented with $20 \mu \mathrm{M}$ 2mercaptoetharol, $300 \mu \mathrm{g} / \mathrm{ml}$ glutamine and $60 \mu \mathrm{g} / \mathrm{ml}$ gentamicin. 


\section{Cytokines}

Murine recombinant IFN- $\gamma$ was obtained as a gift from Pittsburgh Cancer Institute. Human recombinant IFN- $\gamma$ was obtained from Genzyme (Cambridge, MA).

\section{Monoclonal antibodies and antibody conjugates}

Supernatants from hybridoma cell lines B468 (secreting anti-H-2Kk), B896 (secreting anti-H-2K ${ }^{b}$ ) and HB95 (secreting anti-HLA-A, B and C ) were used as sources of monoclonal antibodies. Hybridoma cell lines B486 and B896 were kindly provided by Dr. Eli Gorelik of the Pittsburgh Cancer Institute and hybridoma HB95 was obtained from ATCC (Rockville, MD, USA). Goat antimouse Ig-FITC from Gibco BRL was used as second antibody in flow cytometric studies.

\section{Tumor cell lines}

Murine tumor cell lines EL-4 (haplotype $\mathrm{H}-2^{\mathrm{b}}$, from ATCC, TIB39) and BW5147 (haplotype $\mathrm{H}-2^{\mathrm{k}}$, from ATCC, TIB47) are $\mathrm{T}$ cell lymphomas originally obtained from $\mathrm{NIA}, \mathrm{NIH}$ (courtesy of Dr. W. H. Adler). Human tumor cell lines, K562, a chronic myelogenous leukemia (ATCC, CCL 243) was also obtained from NIA, NIH, and HR7, a gastric carcinoma cell line was originally obtained from Pittsburgh Cancer Institute (courtesy of Dr. T. L. Whiteside).

\section{Preparation of MHC-AF}

Human MHC-AF was purified from the culture supernatant of human peripheral blood lymphocytes (HPBLs) activated by concanavalin A (Con-A), as described before (Saxena et al., 1996; Raval et al., 1997). Mouse MHC-AF preparations were derived from culture supernatants of mouse spleen cells activated in a mixed lymphocyte reaction (Saxena et al., 1981), by using the purification scheme utilized for rat MHC-AF (Saxena et al., 1988). Human MHC-AF has previously been shown to lack any IFN like anti-viral activity (Saxena et al., 1996). Murine MHCAF preparations were also devoid of any anti-viral activity assessed by using a L-cells VSV system of IFN bioassay (Saxena et al., 1988).

\section{Estimation of cell surface antigen on tumor cell lines}

The tumor cells in culture were treated with IFN- $\gamma(500$ $\mathrm{U} / \mathrm{ml}$ ) or MHC-AF preparations $(50 \mu \mathrm{g} / \mathrm{ml})$ for 2 to 3 day, and stained for class I MHC antigens as described before (Saxena et al., 1988, 1996; Raval et al., 1997). Briefly, control or treated tumor cells were suspended in $50 \mu \mathrm{l}$ of hybridoma supernatants containing relevant anti-MHC class I antibodies and incubated for $20 \mathrm{~min}$ at $4^{\circ} \mathrm{C}$. Cells were washed once with cold phosphate-buffered saline (PBS) and suspended in $50 \mu$ lo goat anti-mouse immunoglobulin FITC at 1:400 dilution. After $20 \mathrm{~min}$. incubation at $4^{\circ} \mathrm{C}$, cells were washed once with cold PBS and fixed in $0.5 \mathrm{ml}$ of $1 \%(\mathrm{w} / \mathrm{v})$ paraformaldehyde in PBS. Stained and fixed cells were analyzed in a Coulter Epics- $X L$ flow

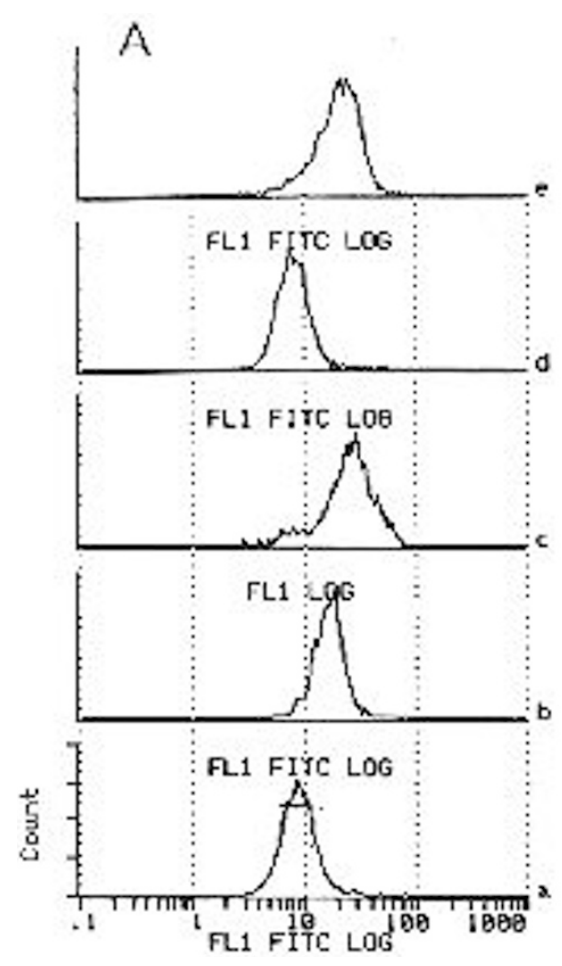

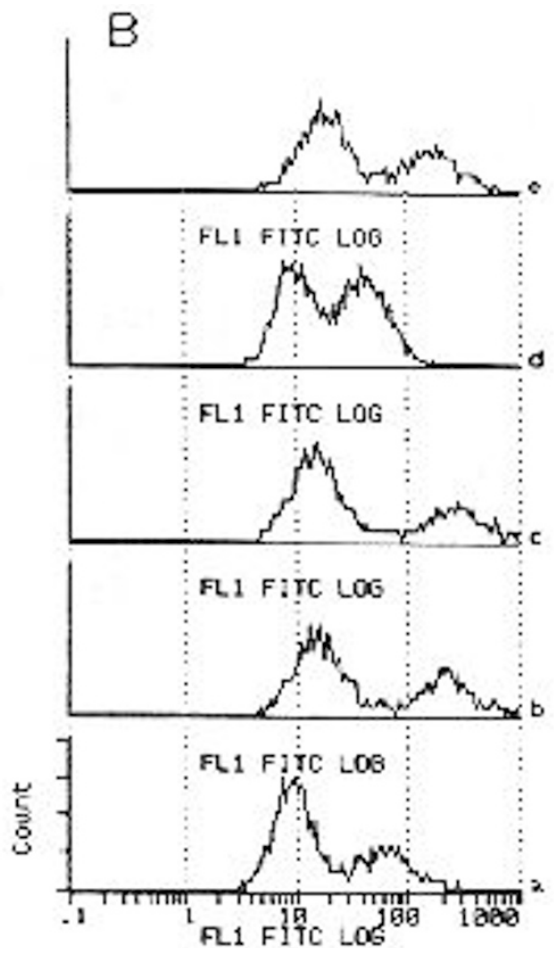

Figure 1. Effect of murine and human MHC-AF and IFN- $\gamma$ on MHC I expression of murine tumor cell lines. Two murine cell lines, EL-4 (Panel A) and BW5147 (Panel B) were cultured alone (a) or with mouse MHC-AF (b), or mouse IFN- $\gamma(\mathrm{c})$, or human IFN- $\gamma$ (d), or human MHC-AF (e) for $48 \mathrm{~h}$. After two days, tumor cells were harvested, stained for class I MHC antigen expression as described in Materials and Methods, and analyzed on a flow cytometer. 

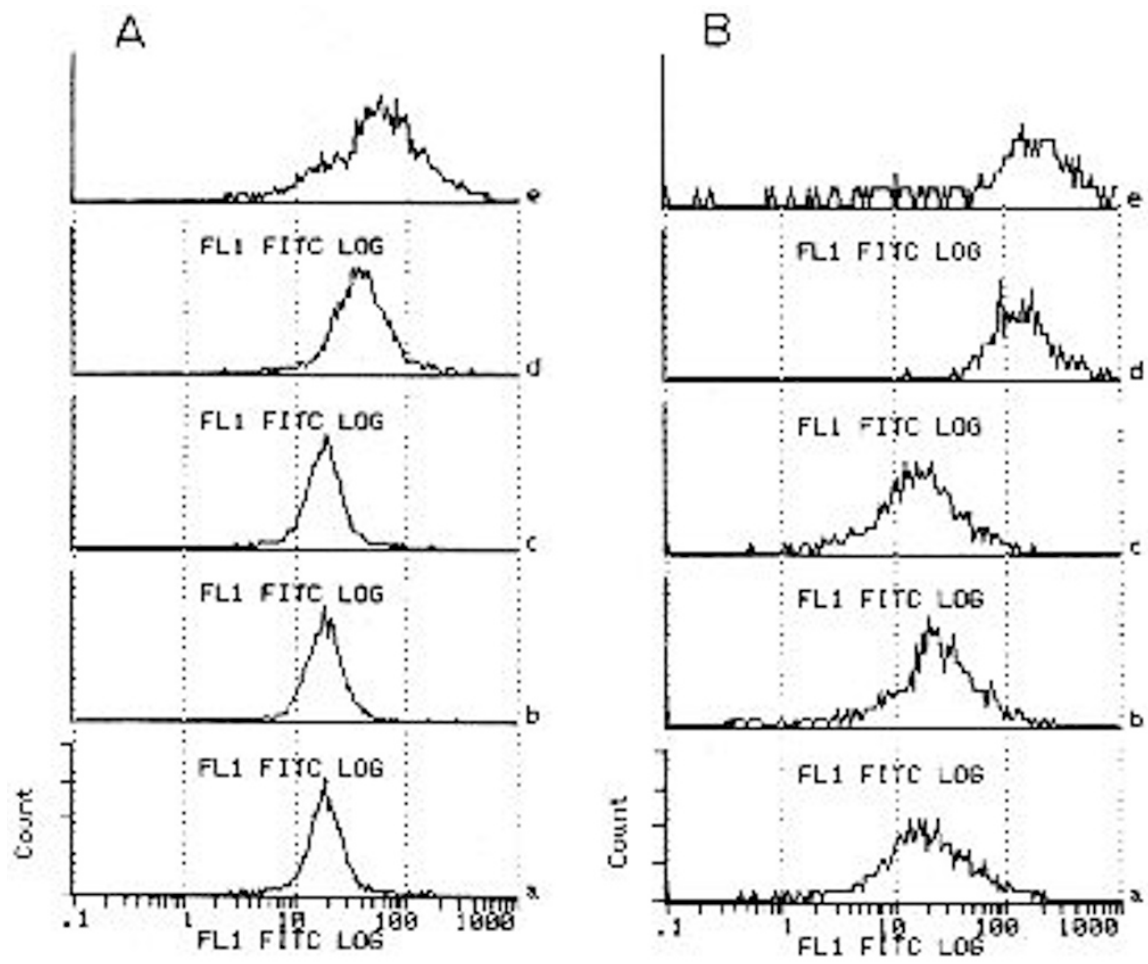

Figure 2. Effect of murine and human MHC-AF and IFN- $\gamma$ on MHC I expression of human tumor cell lines. Two human cell lines, K562 (Panel A) and HR7 (Panel B) were cultured alone (a) or with mouse MHC-AF (b), or mouse IFN- $\gamma$ (c), or human IFN- $\gamma(\mathrm{d})$, or human MHC-AF (e) for 48 h. After two days, tumor cells were harvested, stained for class I MHC antigen expression as described in Materials and Methods, and analyzed on a flow cytometer.

\section{cytometer.}

\section{Results}

\section{Effect of recombinant IFN- $\gamma$ and MHC-AF on the expression of MHC class I antigens on two murine tumor cell lines}

EL4 and BW5147 murine cell lines were treated with mouse or human IFN- $\gamma$ or with human or mouse MHC-AF preparations for two days, and stained for expression of MHC class I antigens. Results of flow cytometric analysis in Figure 1 show that mouse IFN- $\gamma$ induced significant increase in the expression of class I MHC antigens on both mouse tumor lines, whereas human IFN- $\gamma$ failed to have any effect on MHC I expression. Both human as well as mouse MHC-AF however increased MHC class I antigen expression on both tumor cell lines.

\section{Effect of recombinant IFN- $\gamma$ and MHC-AF on the expression of MHC class I antigens on two human tumor cell lines}

K562 and HR7 human tumor cell lines were treated with mouse or human IFN- $\gamma$ or with human or mouse MHC-AF preparations for two days, and stained for expression of $\mathrm{MHC}$ class I antigens. Results of flow cytometric analysis in Figure 2 show that mouse IFN- $\gamma$ as well as mouse MHC-AF had no effect on the expression of class I MHC antigens on both K562 and HR7 tumor lines. Human IFN- $\gamma$ as well as human MHC-AF, however, increased $\mathrm{MHC}$ class I antigen expression on both tumor cell lines.

These results indicate that the biological activity of
IFN- $\gamma$ is strictly species specific since mouse and human IFN- $\gamma$ acted on mouse and human cell lines only. In case of MHC-AF, however, mouse MHC-AF was species specific in action whereas human MHC-AF was biologically active on both mouse and human cell lines.

\section{Discussion}

We have previously reported partial purification and characterization of a rat MHC-AF (Saxena, 1987; Saxena et al., 1988). The rat factor is a 12-kDa protein with an isoelectric $\mathrm{pH}$ of 4.8 (Saxena et al., 1988). Recently, partial purification and characterization of human MHCAF has also been reported and it appears to be a 30kDa protein with pl of 6.0 (Saxena et al., 1996; Raval et al., 1997). Using the purification scheme standardized for rat $\mathrm{MHC}-\mathrm{AF}$, we have now been able to partially purify a mouse MHC-AF from culture supernatants of mouse spleen cells activated in mixed lymphocyte reaction (Puri, 1995). Human and mouse MHC-AF are assayed by their effect on the expression of MHC class I antigens on human and mouse tumor cell lines respectively. Since IFN- $\gamma$ is also active in MHC-AF assays and activated T lymphocytes (e.g. in mitogen activated spleen cells and in mixed lymphocyte reaction) produce IFN- $\gamma$, that raises the question whether MHC-AF is identical to IFN- $\gamma$ or somehow related to IFN- $\gamma$. Based upon biological, immunological and biochemical characterization of $\mathrm{MHC}$ AF preparations, we feel convinced that MHC-AF is a new cytokine distinct from IFN- $\gamma$ (Saxena, 1987; Saxena 
et al., 1988, 1989, 1992, 1996; Puri, 1995; Raval et al., 1997). Final confirmation of a distinct identity of MHCAF wll come when this factor is purified to homogeniety and its structure determined. Since MHC-AF is produced in extremely small quantities, its final purification is yet to be achieved. In our present study, we have used partially purified MHC-AF preparations from mouse and human sources. These MHC-AF preparations were, however, devoid of any IFN- $\gamma$ contamination as assessed by anti-viral bioassay of IFN. We tested for the augmentation of MHC class I expression on two mouse and two human tumor cell lines in response to IFN- $\gamma$ and MHC$\mathrm{AF}$ from the two species. While both mouse and human IFN- $\gamma$ and mouse MHC-AF were strictly species specific in their biological activities, human MHC-AF acted in a species non-specific way, since it increased the MHC class I expression on both mouse and human cell lines. Differences in the species specificity patterns of MHC$\mathrm{AF}$ and IFN- $\gamma$ provide an important fresh evidence which indicate that the two mediators are distinct from each other.

\section{Acknowledgment}

This work was supported by a DBT grant to R.K.S.

\section{References}

Dokhelar, M., Testa, U., Vainchenkar, W., Finale, V., Tetlaud, C., Salem, P. and Tursz, T. (1982) NK cell sensitivity of the leukemic K562: Effect of sodium butyrate and hemin induction. J. Immunol. 128: 211-216

Dokhelar, M., Crrosson, D., Wakasugi, H., Tabilio, A., Testa, U., Vainchenkar, W. and Tursz T. (1984) K562 cells induced to differentiate by phorbol tumor promoters resist NK Iysis. Cell. Immunol. 87: 389-399

Karre, K., Ljunggren, H. G., Piontek, G. and Kiessling, R. (1986) Selective rejection of H2-deficient lymphoma variants suggests alternative immune defense strategy. Nature 319: 678

Piontek, G. E., Taniguchi, K., Ljunggren, H. G., Greenberg, A., Kiessling, R., Klein, G. and Karre, K. (1985) Yac-1 MHC class 1 variants reveal an association between decreased NK sensitivity and increased $\mathrm{H}-2$ expres-sion after IFN treatment or in vivo passage. J. Immunol. 135: 4288

Puri, N. (1995) Purification and characterization of two novel putative cytokines released by activated mouse spleen cells. Ph. D. Thesis, Jawaharlal Nehru University, New Delhi, India

Raval, A., Puri, N. and Saxena, R. K. (1997) Generation of human class I major histocompatibility complex activating factor (MHC-AF) in serum free medium and its partial characterization. J. Biosciences 22: 59-68

Saxena, R. K. (1997) Ontogeny of inhibitory receptors for MHC mole-cules on NK cells. Immunol. Today 18: 146

Saxena, R. K., Saxena, Q. B. And Adler, W.H. (1981) Defective T-cell response in beige mutant mice. Nature 295: 24-25

Saxena, R. K. (1987) A spleen cell derived factor imparts resistance to NK cell mediated lysis in a mouse lymphoma cell line. Immunol. Lett. 15: 105-108

Saxena, R. K., Saxena, Q. B. and Adler, W. H. (1988) Properties and characterization of a rat spleen cell derived factor which produces resistance to NK cell lysis in YAC lymphoma cells. J. Immunol. 141: 1782-1787

Saxena, R. K., Chrest, F. J. and Adler, W. H. (1989) Modulation of major histocompatibility antigens and inhibition of proliferation activity of YAC lymphoma cells by natural killer-resistance inducing factor. Nat. Immun. Cell Growth Regul. 8: 197-208

Saxena, R. K., Saxena, Q. B., Sarin, A. and Herberman, R. B. (1992) Natural killerresistance inducing factor (NK-RIF): A new immuno-modulatory cytokine. In Natural Killer Cells: Receptors, Signalling and Mechanisms (Lotzova, E. and Herberman, R. B., eds), pp. 381-392, CRC Press, New York

Saxena, R. K., Saxena, Q. B., Whiteside, T. L., Goldfarb, R. H. and Herberman, R. B. (1996) Partial purification and characterization of a novel human factor that augments the expression of class I MHC antigens on tumor cells. J. Biosciences 21 : 13-25

Wiebke, E. A., Custer, M. C., Rosenberg, S. A. and Lotze M. T. (1990) Cytokines alter target cell susceptibility to lysis: Evaluation of non major histocompatibility complex effectors reveal differentiated effect on natural and lymphokine activated killing. J. Biol. Resp. Modi. 9: 113-126

Zinkernagel, R. M. and Doherty, P. C. (1979) MHC-restricted cytotoxic T cells studies on the biological role of polymorphic major transplantation antigens determining T-Cell restriction-specificity, function and respon-siveness. Adv. Immunol. 27: 51-177 